\title{
Optimization of annealing of dopant to increase sharpness of $p-n$ junctions in a heterostructure with drain of dopant
}

\author{
E. L. Pankratov • E. A. Bulaeva
}

Received: 25 March 2013/Accepted: 25 April 2013/Published online: 15 May 2013

(c) The Author(s) 2013. This article is published with open access at Springerlink.com

\begin{abstract}
It has been recently shown, that manufacturing of $p-n$ junctions by dopant diffusion or ion implantation in heterostructures and optimization of annealing time leads to increasing of their sharpness and homogeneity of dopant distribution in enriched area. In this paper, we consider influence of defects of doped structure (mismatch dislocations and similar), which became as drain of atoms of dopant, on dopant distribution in diffusive-junction rectifier.
\end{abstract}

Keywords Diffusion-heterojunction rectifier · Modeling of dopant diffusion - Accounting drain of dopant .

Optimization of annealing of dopant

\section{Introduction}

In the present time, elaboration of new devices of solid state electronic devices is intensively done. The second way for intensive elaboration is refinement of characteristics of traditional solid state electronic devices (EsmaeiliRad et al. 2007; Huang et al. 2003; Lai et al. 2007; Kitada et al. 2009; Lei et al. 2009; Volocobinskaya et al. 2001; Vasil'ev et al. 2002). One of the questions of the refinement is increasing of sharpness of $p-n$ junctions and manufacturing the devices as more shallow (Andronov

\section{E. L. Pankratov $(\bowtie)$}

Nizhny Novgorod State University, 23 Gagarin avenue,

Nizhny Novgorod 603950, Russia

e-mail: elp2004@mail.ru

\section{E. A. Bulaeva}

Nizhny Novgorod State University of Architecture and Civil Engineering, 65 Il'insky street, Nizhny Novgorod 603950, Russia

e-mail: hellen-bulaeva@yandex.ru et al. 1998; Sisiyany et al. 2002). One way to increase sharpness of $p-n$ junction is using laser annealing (Varonina et al. 1999; Pankratov 2005). The second one is using inhomogeneity of heterostructure $(\mathrm{H})$ (Kelleher et al. 2009; Pankratov 2010, 2012). It could be used as another standard of approach. In this paper, we consider an approach to increase sharpness of diffusive-junction rectifier using defects of doped structure.

In this paper, we consider a $\mathrm{H}$ with two layers (see Fig. 1). One layer is a substrate (S). The second one is an epitaxial layer (EL). A dopant has been infused in the EL. One can obtain increasing of sharpness of the diffusivejunction rectifier and at the same time increasing of homogeneity of dopant distribution in the rectifier after that, when dopant achieves the interface between layers of $\mathrm{H}$ (see Fig. 2). Recently it has been shown, that after annealing of dopant with optimal value of annealing time $\Theta$ compromise between increasing of sharpness of $p$ $n$ junction and increasing of homogeneity of dopant distribution in the rectifier could be achieved (Pankratov 2005, 2010, 2012). Main aim of the present paper is taking into account drains of atoms of dopant on defects of doped structure (mismatch dislocations and similar).

\section{Method of solution}

Redistribution of dopant has been described by the second Fick's law (Shalimova 1985; Gotra 1991; Alexandrov 2002)

$$
\begin{aligned}
\frac{\partial C(x, t)}{\partial t}= & \frac{\partial}{\partial x}\left[D_{\mathrm{C}} \frac{\partial C(x, t)}{\partial x}\right]-k_{\mathrm{R}}(x, T) C(x, t) \\
& +k_{\mathrm{G}}(x, T) C(x, t) .
\end{aligned}
$$

Here $C(x, t)$ is the spatiotemporal distribution of dopant; $D_{\mathrm{C}}$ is the dopant diffusion coefficient; $k_{\mathrm{R}}(x, T)$ is the 


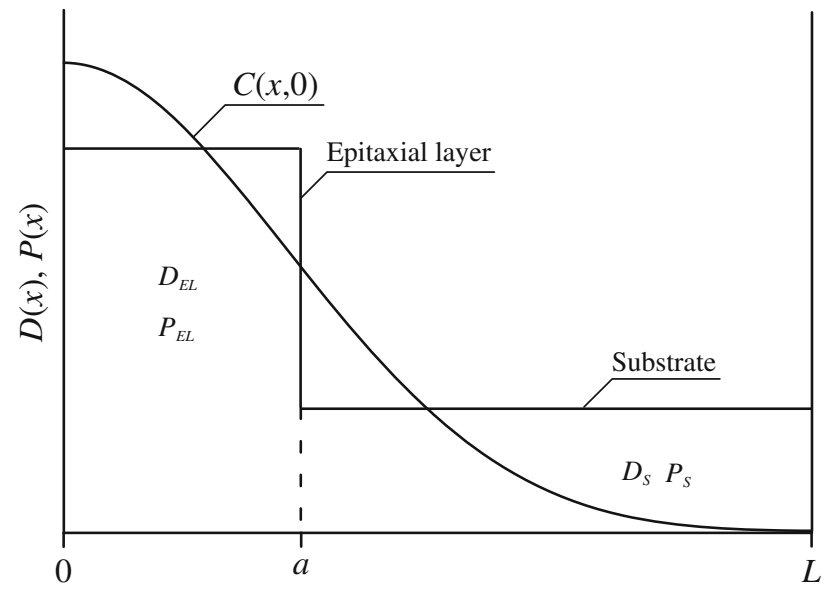

Fig. 1 Heterostructue with epitaxial layer and substrate

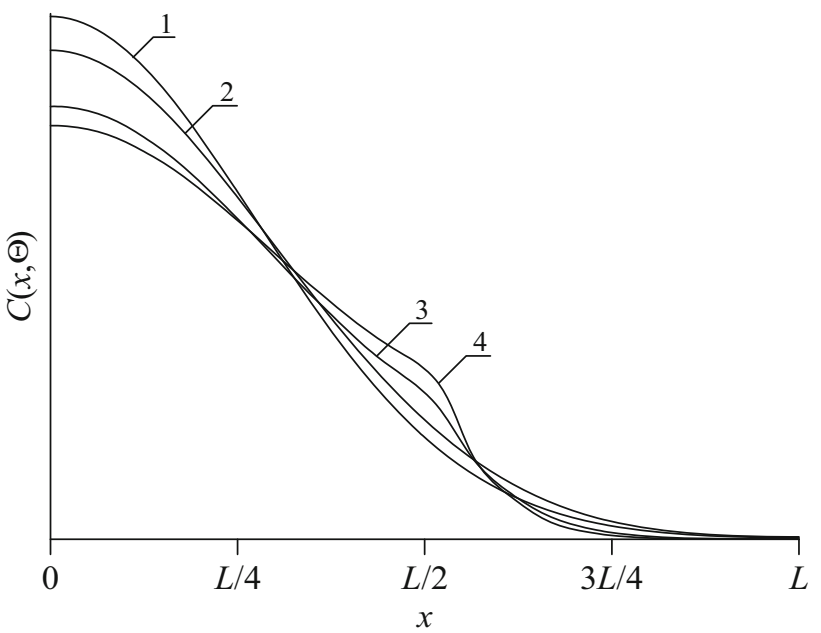

Fig. 2 Dopant distributions for different values of annealing time. Increasing of number of curves corresponds to increasing of value of annealing time. Interface between layers coincides with midpoint of the heterostructure, i.e., $x=L / 2$

parameter, which characterize speed of capturing of atoms of dopant by drains; $k_{\mathrm{G}}(x, T)$ is the parameter, which characterize speed of returning of atoms of dopant from drains. We transform the Eq. (1) to the following form by combination of two last terms into one

$\frac{\partial C(x, t)}{\partial t}=\frac{\partial}{\partial x}\left[D_{\mathrm{C}} \frac{\partial C(x, t)}{\partial x}\right]-K(x, T) C(x, t)$,

where $K(x, T)=k_{\mathrm{R}}(x, T)-k_{\mathrm{G}}(x, T)$. The Eq. (1) is complemented by the following boundary and initial conditions $\left.\frac{\partial C(x, t)}{\partial x}\right|_{x=0}=\left.\frac{\partial C(x, t)}{\partial x}\right|_{x=L}=0, C(x, 0)=f_{\mathrm{C}}(x)$.

The conditions have been written in the most common form. However dopant usually did not achieves the boundary $x=L$ of $\mathrm{H}$. In this situation, appropriate boundary condition could be written as: $C(L, t)=0$.

Value of diffusion coefficient $D_{\mathrm{C}}$ depends on properties of materials of $\mathrm{H}$, velocities of heating and cooling (with account Arrhenius law) of the materials and spatiotemporal distribution of dopant concentration. The last dependence could be approximated by the following function (Gotra 1991)

$D_{\mathrm{C}}=D_{L}(x, T)\left[1+\xi \frac{C^{\gamma}(x, t)}{P^{\gamma}(x, T)}\right]$.

Here $D_{L}(x, T)$ is spatial (due to inhomogeneity of $\mathrm{H}$ ) and temperature (due to Arrhenius law, where $T$ is the temperature) dependences of diffusion coefficient; $P(x, T)$ is the limit of solubility of dopant; parameter $\gamma$ depends on properties of materials and could be integer in the interval $\gamma \in[1,3]$ (Gotra 1991). Concentrational dependence of diffusion coefficient is discussed in detail in (Gotra 1991).

To determine spatiotemporal distribution of dopant concentration let us use the method of averaging of function correction (Pankratov 2010; Alexandrov 2002). To use the approach we transform the Eq. (1a) to the following integral form

$$
\begin{aligned}
C(x, t)= & C(x, t)+\frac{1}{L^{2}}\left\{\int_{0}^{t} D_{L}(x, T)\left[1+\xi \frac{C^{\gamma}(x, \tau)}{P^{\gamma}(x, T)}\right]\right. \\
& \times C(x, \tau) d \tau-\int_{0}^{t} \int_{0}^{x} K(v, T) C(v, \tau)(x-v) \mathrm{d} v \mathrm{~d} \tau \\
& -\int_{0}^{t} \int_{0}^{x} C(v, \tau)\left[1+\xi \frac{C^{\gamma}(v, \tau)}{P^{\gamma}(v, T)}\right] \frac{\partial D_{L}(v, T)}{\partial v} \mathrm{~d} v \mathrm{~d} \tau \\
& +\int_{0}^{t} \int_{0}^{L} C(x, \tau)\left[1+\xi \frac{C^{\gamma}(x, \tau)}{P^{\gamma}(x, T)}\right] \frac{\partial D_{L}(x, T)}{\partial x} \mathrm{~d} x \mathrm{~d} \tau \\
& +\int_{0}^{t} \int_{0}^{L}(L-x) K(x, T) C(x, \tau) \mathrm{d} x \mathrm{~d} \tau+\int_{0}^{L}(L-x) C(x, t) \mathrm{d} x \\
& \left.-\int_{0}^{x}(x-v) \times C(v, t) \mathrm{d} v-\int_{0}^{L}(L-x) f(x) \mathrm{d} x+\int_{0}^{x}(x-v) f(v) \mathrm{d} v\right\} .
\end{aligned}
$$

To determine the first-order approximation $C_{1}(x, t)$ of dopant concentration let us replace determining function $C(x, t)$ in the right side of Eq. (1b) on its average value $\alpha_{1}$. After the replacement one can obtain the following relation for the first-order approximation $C_{1}(x, t)$ of dopant concentration in the following form 


$$
\begin{aligned}
C_{1}(x, t)= & \alpha_{1}+\frac{1}{L^{2}}\left\{\alpha_{1} \int_{0}^{t} D_{L}(x, T)\left[1+\frac{\xi \alpha_{1}^{\gamma}}{P^{\gamma}(x, T)}\right] \mathrm{d} \tau\right. \\
& -\alpha_{1} \int_{0}^{t} \int_{0}^{x}(x-v) K(v, T) \mathrm{d} v \mathrm{~d} \tau-\alpha_{1} \\
& \times \int_{0}^{t} \int_{0}^{x}\left[1+\frac{\xi \alpha_{1}^{\gamma}}{P^{\gamma}(v, T)} \frac{\partial D_{L}(v, T)}{\partial v} \mathrm{~d} v \mathrm{~d} \tau\right. \\
& +\alpha_{1} \int_{0}^{t} \int_{0}^{L}(L-x) K(x, T) \mathrm{d} x \mathrm{~d} \tau+\int_{0}^{t} \int_{0}^{L} \frac{\partial D_{L}(x, T)}{\partial x} \\
& \times \alpha_{1}\left[1+\frac{\xi \alpha_{1}^{\gamma}}{P^{\gamma}(x, T)}\right] \mathrm{d} x \mathrm{~d} \tau+\frac{\alpha_{1}}{2}\left(L^{2}-x^{2}\right) \\
& \left.-\int_{0}^{L}(L-x) f(x) \mathrm{d} x+\int_{0}^{x}(x-v) f(v) \mathrm{d} v\right\}
\end{aligned}
$$

The average value $\alpha_{1}$ of the function $C_{1}(x, t)$ could be determined by the following standard relation

$\alpha_{i}=\frac{1}{L \Theta} \int_{0}^{\Theta} \int_{0}^{L} C_{i}(x, t) \mathrm{d} x \mathrm{~d} t$,

where $\Theta$ is the observation time on diffusion process. Integration of the function $C_{1}(x, t)$ with account relation (4) leads to the following equation for the average value $\alpha_{1}$, which depends on parameter $\gamma$

$$
\begin{aligned}
& \alpha_{1} \int_{0}^{\Theta}(\Theta-t) \int_{0}^{L} D_{L}(x, T)\left[1+\frac{\xi \alpha_{1}^{\gamma}}{P_{\gamma}^{\gamma}(x, T)}\right] \mathrm{d} x \mathrm{~d} t \\
& -\frac{\alpha_{1}}{2} \int_{0}^{\Theta}(\Theta-t) \int_{0}^{L}\left(L^{2}-x^{2}\right) K(x, T) \mathrm{d} x \mathrm{~d} t \\
& -\alpha_{1} \int_{0}^{\Theta}(\Theta-t) \int_{0}^{L}(L-x)\left[1+\frac{\xi \alpha_{1}^{\gamma}}{P^{\gamma}(x, T)}\right] \frac{\partial D_{L}(x, T)}{\partial x} \mathrm{~d} x \mathrm{~d} t \\
& +\alpha_{1} \int_{0}^{\Theta} \int_{0}^{L}(L-x) K(x, T) \mathrm{d} x(\Theta-t) \mathrm{d} \tau \\
& +\alpha_{1} \int_{0}^{\Theta}(\Theta-t) \int_{0}^{L}\left[1+\frac{\xi \alpha_{1}^{\gamma}}{P^{\gamma}(x, T)}\right] \frac{\partial D_{L}(x, T)}{\partial x} \mathrm{~d} x \mathrm{~d} \tau \\
& +\int_{0}^{L}\left(L^{2}-x^{2}\right) f(x) \mathrm{d} x \frac{\Theta}{2}+L^{3} \Theta \frac{\alpha_{1}}{3}-\Theta L \int_{0}^{L}(L-x) f(x) \mathrm{d} x=0 .
\end{aligned}
$$

The second-order approximation of dopant concentration $C_{2}(x, t)$ could be determined by standard iteration procedure of method of averaging of function correction (Pankratov 2010; Sokolov 1955), i.e., by replacement the determining function $C(x, t)$ in the right side of the Eq. (1b) on sum of average value $\alpha_{2}$ of the second-order approximation of dopant concentration $C_{2}(x, t)$ and the first-order approximation of dopant concentration $C_{1}(x, t)$. In this case, the second-order approximation could be written as

$$
\begin{aligned}
& C_{2}(x, t)=\alpha_{2}+C_{1}(x, t)+\frac{1}{L^{2}}\left(\int_{0}^{t} D_{L}(x, T)\right. \\
& \times\left[\alpha_{2}+C_{1}(x, \tau)\right]\left\{1+\xi \frac{\left[\alpha_{2}+C_{1}(x, \tau)\right]^{\gamma}}{P^{\gamma}(x, T)}\right\} \mathrm{d} \tau \\
& -\int_{0}^{t} \int_{0}^{x}(x-v) K(v, T)\left[\alpha_{2}+C_{1}(v, \tau)\right] \mathrm{d} v \mathrm{~d} \tau \\
& +\int_{0}^{t} \int_{0}^{L} K(x, T)(L-x)\left[\alpha_{2}+C_{1}(x, \tau)\right] \mathrm{d} x \mathrm{~d} \tau \\
& +\int_{0}^{t} \int_{0}^{L}\left[C_{1}(x, \tau)+\alpha_{2}\right]\left\{1+\xi \frac{\left[\alpha_{2}+C_{1}(x, \tau)\right]^{\gamma}}{P^{\gamma}(x, T)}\right\} \\
& \times \frac{\partial D_{L}(x, T)}{\partial x} \mathrm{~d} x \mathrm{~d} \tau-\int_{0}^{t} \int_{0}^{x} \frac{\partial D_{L}(v, T)}{\partial v} \\
& \times\left\{1+\xi \frac{\left[\alpha_{2}+C_{1}(v, \tau)\right]^{\gamma}}{P^{\gamma}(v, T)}\right\}\left[\alpha_{2}+C_{1}(v, \tau)\right] \mathrm{d} v \mathrm{~d} \tau \\
& -\int_{0}^{L}(L-x) f(x) \mathrm{d} x+\int_{0}^{L}(L-x)\left[\alpha_{2}+C_{1}(x, t)\right] \mathrm{d} x \\
& \left.-\int_{0}^{x}(x-v)\left[\alpha_{2}+C_{1}(v, t)\right] \mathrm{d} v+\int_{0}^{x}(x-v) f(v) \mathrm{d} v\right) .
\end{aligned}
$$

Average value $\alpha_{2}$ of the second-order approximation of dopant concentration could be determined by the standard relation (4). After substitution of the function $C_{2}(x, t)$ in the relation (4), we obtain the equation for the average value $\alpha_{2}$ in the following form

$$
\begin{aligned}
& \int_{0}^{\Theta}(\Theta-t) \int_{0}^{L} D_{L}(x, T)\left[\alpha_{2}+C_{1}(x, t)\right]\left\{1+\xi \frac{\left[\alpha_{2}+C_{1}(x, t)\right]^{\gamma}}{P^{\gamma}(x, T)}\right\} \mathrm{d} x \mathrm{~d} t \\
& -\frac{1}{2} \int_{0}^{\Theta}(\Theta-t) \int_{0}^{L} K(x, T)\left(L^{2}-x^{2}\right)\left[\alpha_{2}+C_{1}(x, t)\right] \mathrm{d} x \mathrm{~d} t \\
& +L \int_{0}^{\Theta} \int_{0}^{L}\left[\alpha_{2}+C_{1}(x, t)\right]\left\{1+\xi \frac{\left[\alpha_{2}+C_{1}(x, t)\right]^{\gamma}}{P^{\gamma}(x, T)}\right\} \frac{\partial D_{L}(x, T)}{\partial x} \mathrm{~d} x \\
& \times(\Theta-t) \mathrm{d} t+L \Theta \int_{0}^{\Theta} \int_{0}^{L}(L-x)\left[\alpha_{2}+C_{1}(x, t)\right] \mathrm{d} x \mathrm{~d} t
\end{aligned}
$$




$$
\begin{aligned}
& -L \int_{0}^{\Theta}(\Theta-t) \int_{0}^{L}(L-x)\left\{1+\xi \frac{\left[\alpha_{2}+C_{1}(v, t)\right]^{\gamma}}{P^{\gamma}(v, T)}\right\} \\
& \times\left[\alpha_{2}+C_{1}(v, t)\right] \frac{\partial D_{L}(v, T)}{\partial v} \mathrm{~d} v \mathrm{~d} t+L \int_{0}^{\Theta}(\Theta-t) \int_{0}^{L}(L-x) \\
& {\left[\alpha_{2}+C_{1}(x, t)\right] K(x, T) \mathrm{d} x \mathrm{~d} t+\frac{\Theta}{2} \int_{0 x}^{L} f(x)\left(L^{2}-x^{2}\right) \mathrm{d} x} \\
& -\Theta L \int_{0}^{L}(L-x) f(x) \mathrm{d} x-\frac{1}{2} \int_{0}^{\Theta} \int_{0}^{L}(L-x)\left[\alpha_{2}+C_{1}(x, t)\right] \mathrm{d} x \mathrm{~d} t=0 .
\end{aligned}
$$

Analysis of spatiotemporal distribution of dopant concentration has been done analytically using the secondorder approximation framework method of averaging of function correction and has been amended numerically.

\section{Discussion}

In this section, we analyzed influence of drains of atoms of dopant on distribution of their concentration in diffusionjunction rectifier. Some distributions of dopant, which corresponds to the rectifier, are present in Fig. 3. In this case drain of the atoms is presented on the right side of the heterostructure. Increasing of number of curve corresponds to increasing of value of annealing time. The figure shows, that availability of drain leads to decreasing of sharpness of the rectifier. Analogous conclusion could be done in the case, when drain of atoms of dopant is presented on the interface between layers of $\mathrm{H}$. The decreasing of sharpness could be though partially compensated using inhomogeneity of heterostructure and nonlinearity of diffusion process. The nonlinearity leads to large influence, when level of doping of materials is high (Pankratov 2005).

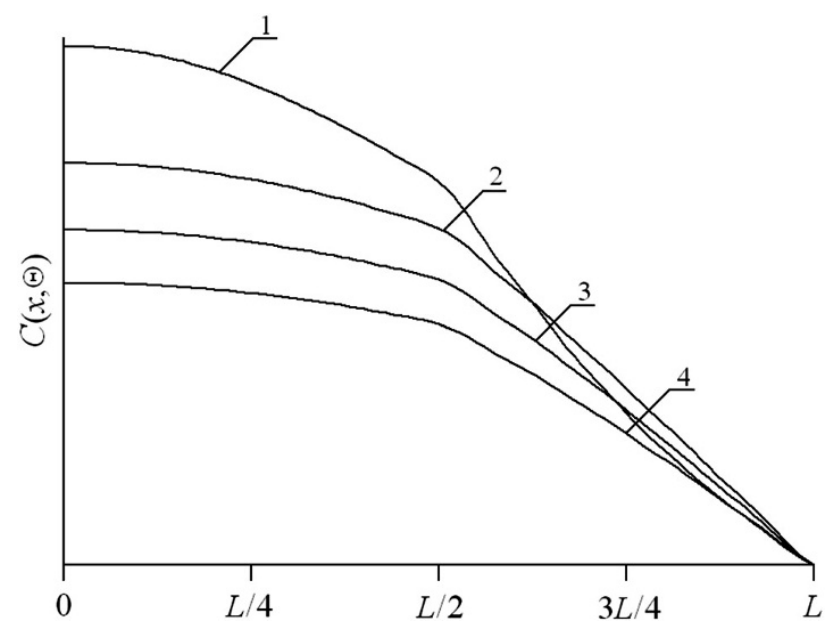

Fig. 3 Normalized distributions of dopant for the case, when drain of atoms of dopant is localized on the right boundary of heterostructure

\section{Conclusion}

In this paper, we analyzed influence of drain of atoms of dopant on distribution of their concentration in diffusionjunction rectifier. It has been shown, that availability of drain leads to decreasing sharpness of $p-n$ junction. It has been considered abilities of compensation of the decreasing.

Acknowledgments This work is supported by the contract 11.G34.31.0066 of the Russian Federation Government and educational fellowship of President of Russia.

Open Access This article is distributed under the terms of the Creative Commons Attribution License which permits any use, distribution, and reproduction in any medium, provided the original author(s) and the source are credited.

\section{References}

Alexandrov OV (2002) Influence of sinks for intrinsic point defects on phosphorus diffusion in silicon. Semiconductors 36(11): $1345-1350$

Andronov AN, Bagraev NT, Klyachkin LE, Robozerov SV (1998) Ultrashallow $p^{+}-n$-junctions in silicon (100): electron-beam diagnostics of sub-surface region. Semiconductors 32(2): 137-144

Esmaeili-Rad MR, Li F, Sazonov A, Nathan A (2007) Stability of nanocrystalline silicon bottom-gate thin film transistors with silicon nitride gate dielectric. J Appl Phys 102(6):064512064518

Gotra ZYu (1991) Technology of microelectronic devices. Radio and Communication, Moscow

Huang J, Blochwitz-Nimoth J, Pfeiffer M, Leo K (2003) Influence of the thickness and doping of the emission layer on the performance of organic light-emitting diodes with PiN structure. J Appl Phys 93(2):838-844

Kelleher EJR, Travers JC, Sun Z, Rozhin AG, Ferrari AC, Popov SV, Taylor JR (2009) Nanosecond-pulse fiber lasers mode-locked with nanotubes. Appl Phys Lett B 95(7):111108-111110

Kitada T, Tanaka F, Takahashi T, Morita K, Isu T (2009) GaAs/AlAs coupled multilayer cavity structures for terahertz emission devices. Appl Phys Lett 95(4):111106-111108

Lai SL, Chan MY, Fung MK, Lee CS, Lee ST (2007) Copper hexadecafluorophthalocyanine and copper phthalocyanine as a pure organic connecting unit in blue tandem organic lightemitting devices. J Appl Phys 101(1):014509-124511

Lei Y, Teng F, Hou Y, Lou Z, Wang Y (2009) Verification of $p$ $n$ junctions in polymer light-emitting electrochemical cells via electrical characterization. Appl Phys Lett 95(10):101105101107

Pankratov EL (2005) Influence of spatial, temporal and concentrational dependence of diffusion coefficient on dopant dynamics: optimization of annealing time. Phys Rev B 72(7):075201075208

Pankratov EL (2010) Decreasing of depth of implanted-junction rectifier in semiconductor heterostructure by optimized laser annealing. J Comp Theor Nanosci 7(1):289-295

Pankratov EL (2012) Using serial radiation processing and microwave annealing decrease depth of $p-n$-junction in a semiconductor heterostructure. Appl Nanosci. doi:10.1007/s13204012-0129-1 
Shalimova KV (1985) Physics of semiconductors. Energoatomizdat, Moscow

Sokolov YuD (1955) About determination mechanical stress in hoisting ropes of mines. Appl Mech 1:23

Sisiyany ST, Sisiyany TS, Raylyan SK (2002) Semiconductors. Shallow $p-n$-junctions in Si prepared by pulse photon annealing. V. 36(5):611-617

Varonina TI, Zotova NV, Kizhaev SS, Molchanov SS, Yakovlev YuP (1999) Luminescent properties of InAs layers and $p-n$-structures grown by metalorganic chemical vapor deposition. Semiconductors 33(10):1168-1172
Vasil'ev VV, Kravchenko AF, Mashukov YuP (2002) Field-dependent photosensitivity of MIS In- $\mathrm{SiO}_{2}-\mathrm{Cd}_{0.28}-\mathrm{Hg}_{0.72} \mathrm{Te}$ structures with an opaque field electrode. Semiconductors 36(9):10681071

Volocobinskaya NI, Komarov IN, Matyukhina TV, Rush AA, Falina IV, Yastrebov AS (2001) Investigation of technological processes of manufacturing of the bipolar power high-voltage transistors with a grid of inclusions in the collector region. Semiconductors 35(8):1013-1017 